\title{
Análise da qualidade de vida em acadêmicos de medicina em universidade particular de Sergipe por meio do WHOQOL-bref: uma abordagem sociodemográfica
}

\author{
Quality of life analysis of medical students at a private university in Sergipe through the
} WHOQOL-bref: a sociodemographic approach

Análisis de la calidad de vida de los estudiantes de medicina de una universidad privada de Sergipe a través del WHOQOL-bref: un enfoque sociodemográfico

\section{Resumo}

Objetivo: Analisar a qualidade de vida e aspectos sociodemográficos em acadêmicos de medicina do primeiro, oitavo e décimo segundo período de uma instituição privada de ensino superior em Sergipe. Métodos: Trata-se de um estudo analítico transversal prospectivo envolvendo acadêmicos da área de medicina, em universidade particular com o sistema de ensino Problem Based Learning (PBL). Foram empregados dois questionários, um sociodemográfico e o World Health Organization's Quality of Life (WHOQOL-bref). Resultados: Do total de 126 estudantes a maioria é do sexo feminino, reside com familiares, nunca casou e possuem faixa etária entre 20 e 35 anos. Além disso, grande parte dos estudantes não trabalha e realiza o pagamento próprio das mensalidades. A pior média nos domínios analisados foi o psicológico. No aspecto sociodemográfico foram encontrados valores significativos com médias mais baixas de qualidade de vida nos que moram em república privada, nos maiores de 35 anos, naqueles que têm financiamento do curso e nos que possuem profissão. Conclusão: De acordo com o estudo fica notório que há prejuízo na qualidade de vida dos estudantes de medicina, no que se refere a fatores sociodemográficos, principalmente, idade, moradia, forma de pagamento do curso e trabalho. Dessa forma, é importante que a universidade incentive atividades de lazer e divulgue o Núcleo de Apoio Pedagógico e Psicossocial (NAPPS).

Palavras-chave: Estudantes de medicina; Qualidade de vida; Saúde mental.

\begin{abstract}
Objective: To analyze the quality of life and sociodemographic aspects of medical students in the first, eighth and twelfth period of a private higher education institution in Sergipe. Methods: This is a prospective cross-sectional analytical study involving medical students at a private university with the Problem Based Learning (PBL) teaching system. Two questionnaires were used, one sociodemographic and the World Health Organization's Quality of Life (WHOQOL-bref). Results: Of the total of 126 students, most are female, live with family members, never married and are aged between 20 and 35 years. In addition, most students do not work and pay their own tuition fees. The worst average in the analyzed domains was the psychological. In the sociodemographic aspect, significant values were found, with lower averages of quality of life among those living in a private republic, those over 35 years of age, those who had the course financed and those who had a profession. Conclusion: According to the study, it is clear that there is a loss in the quality of life of medical students, with regard to sociodemographic factors, especially age, housing,
\end{abstract}


form of payment for the course and work. Thus, it is important for the university to encourage leisure activities and publicize the Pedagogical and Psychosocial Support Center (NAPPS).

Keywords: Medical students; Quality of life; Mental health.

\begin{abstract}
Resumen
Objetivo: Analizar la calidad de vida y aspectos sociodemográficos de los estudiantes de medicina del primer, octavo y duodécimo período de una institución privada de educación superior en Sergipe. Métodos: Se trata de un estudio analítico transversal prospectivo que involucra a estudiantes de medicina de una universidad privada con el sistema de enseñanza de Aprendizaje Basado en Problemas (PBL). Se utilizaron dos cuestionarios, uno sociodemográfico y el de Calidad de Vida de la Organización Mundial de la Salud (WHOQOL-bref). Resultados: Del total de 126 estudiantes, la mayoría son mujeres, viven con familiares, nunca se han casado y tienen entre 20 y 35 años. Además, la mayoría de los estudiantes no trabajan y pagan sus propias tasas de matrícula. El peor promedio en los dominios analizados fue el psicológico. En el aspecto sociodemográfico, se encontraron valores significativos, con menores promedios de calidad de vida entre los que viven en una república privada, los mayores de 35 años, los que tenían el curso financiado y los que tenían una profesión. Conclusión: Según el estudio, es evidente que existe una pérdida en la calidad de vida de los estudiantes de medicina, en cuanto a factores sociodemográficos, especialmente la edad, la vivienda, la forma de pago del curso y el trabajo. Por ello, es importante que la universidad fomente el ocio y dé a conocer el Centro de Apoyo Pedagógico y Psicosocial (NAPPS).
\end{abstract}

Palabras clave: Estudiantes de medicina; Calidad de vida; Salud mental.

\title{
1. Introdução
}

O termo qualidade de vida (QV) foi definido pela Organização Mundial da Saúde (OMS) como:

"[...] a percepção do indivíduo de sua posição na vida, no contexto da cultura e sistema de valores nos quais ele vive e em relação aos seus objetivos, expectativas, padrões e preocupações.”(The World Health Organization Quality of Life Assessment (WHOQOL), 1995).

Assim, a QV de acordo com a OMS é tratada como um parâmetro subjetivo que possui aspectos da saúde física, do estado psicológico, das relações sociais e do meio ambiente (The World Health Organization Quality of Life Assessment (WHOQOL), 1995).

No contexto universitário atual, a qualidade de vida dos acadêmicos está diretamente ligada ao desempenho destes no exercício de suas atividades, uma das principais variáveis seria a saúde mental (Meira et al., 2017). A alta carga acadêmica do curso de medicina pode dificultar a qualidade de vida dos estudantes, principalmente quando os alunos participam da área clínica da graduação (Irribarra T. et al., 2018).

Atualmente, também é levado em consideração a importância do suporte social e do ambiente educacional como fatores que interferem na qualidade de vida dos estudantes (Hwang et al., 2017).

Em estudo realizado por Oliveria (2018), com 143 acadêmicos de medicina no âmbito da qualidade de vida. Houve uma percepção ruim na QV global, sendo que o estresse percebido e o domínio da exaustão emocional apresentaram maior associação com tal desfecho. Estes e outros fatores, quando associados, são responsáveis pela redução do entusiasmo dos alunos assim que adentram a universidade. Nota-se que estes vão tornando-se cada vez mais desestimulados e a busca pelo conhecimento se esvai com a rotina (Scholz et al., 2015).

Em outro estudo em que foram avaliados 394 estudantes, os menores escores de QV observados foram para: mulheres, com morbidade crônica referida (MCR), cotistas, e do terceiro e sexto ano (Chazan et al., 2015). Já em outro estudo realizado em uma universidade particular de Salvador, o escore relativo ao estresse percebido ficou acima do valor considerado indicativo de alto nível de estresse e a qualidade de vida foi considerada regular. Além disso, foi relatada má qualidade de sono por $72 \%$ dos alunos e presença de possível distúrbio de sono em 17\% dos acadêmicos (Amorim et al., 2018).

Desse modo, esse estudo foi conduzido para identificar características sociodemográficas que potencialmente influenciam a qualidade de vida dos estudantes de medicina em uma universidade particular de Aracaju, Sergipe, Brasil. 


\section{Metodologia}

\section{Delineamento metodológico}

Trata-se de um estudo analítico transversal prospectivo envolvendo acadêmicos da área de medicina em universidade particular com o sistema de ensino Problem Based Learning (PBL), localizada em Aracaju, capital do estado, na região Nordeste do Brasil. A coleta de dados foi realizada durante quatro meses no ano de 2019.

O presente estudo tem o intuito de investigar a qualidade de vida, através do questionário World Health Organization's Quality of Life (WHOQOL-bref). Esse instrumento apresenta 24 questões, graduadas em escala de zero a cinco pontos, divididas em quatro domínios: físico, psicológico, relações sociais e meio ambiente.

Além deste instrumento de pesquisa foi utilizado um questionário sociodemográfico, o qual tem objetivo de identificar fatores que possam interferir na qualidade de vida dos estudantes de medicina, como: questões psicossociais relacionadas ao financiamento do curso, idade, profissão, sexo, moradia, estado civil, raça/cor, período da graduação.

Para atingir os objetivos da pesquisa foi utilizado o cruzamento das questões dos dois questionários, a fim de observar a qualidade de vida em uma escala de 0 a 100 a partir dos quatro domínios do questionário WHOQOL-bref, no qual quanto maior o valor, melhor a QV.

\section{Amostra}

Foi utilizada uma amostra por conveniência nos dias em que os acadêmicos de medicina estiveram na universidade, sem distinção de sexo. Foram incluídos apenas estudantes maiores de 18 anos de ambos os sexos, do $1^{\circ}, 8^{\circ}$ e $12^{\circ}$ períodos, uma vez que representam diferentes etapas do processo de graduação, são elas: a fase de ingresso no curso ( $1^{\circ}$ período), a etapa que antecede o internato ( $8^{\circ}$ período), e, por fim, o $12^{\circ}$ período (última etapa para conclusão da graduação). Foram utilizados como critérios de exclusão: apresentar idade inferior a 18 anos em ambos os sexos, não estar cursando o primeiro, oitavo, décimo segundo período e não ter respondido aos questionários de forma completa.

\section{Confecção de banco de dados e análise dos resultados}

Os resultados foram apresentados sob a forma de números absolutos, proporções (variáveis categóricas) e média \pm desvio-padrão (variáveis contínuas). Os dados obtidos foram armazenados e tabulados usando as ferramentas dos programas Microsoft Office Excel $\AA$, de propriedade dos pesquisadores. Os resultados foram expressos em termos absolutos, relativos e percentagens, representados através de tabelas.

As variáveis categóricas foram descritas por meio de frequência absoluta e relativa percentual. As variáveis contínuas foram descritas por meio de média e desvio padrão. A hipótese de independência entre variáveis categóricas foi testada por meio dos testes Qui-Quadrado de Pearson e Exato de Fisher. A hipótese de aderência das variáveis contínuas à distribuição normal foi testada por meio do teste de Shapiro-Wilks. Quando confirmada, a hipótese de igualdade de médias foi testada por meio do teste $\mathrm{T}$ para amostras independentes ( 2 grupos) ou ANOVA ( 3 ou mais grupos), caso contrário, foi testada por meio dos testes de Mann-Whitney (2 grupos) ou Kruskal-Wallis (3 ou mais grupos). Foram aplicados os testes Z com correção de Bonferroni para os testes de múltiplas comparações envolvendo variáveis categóricas e o teste de Mann-Whitney-Bonferroni para os testes de múltiplas comparações envolvendo variáveis contínuas. Foi utilizado a correlação de Spearman para variáveis contínuas. O nível de significância adotado foi de 5\% e o software utilizado foi o R Core Team 2020 (Versão 4.0.4). 


\section{Aspectos éticos}

Os pesquisadores garantem sigilo, confiabilidade e fidedignidade acerca dos questionários respondidos. Para tanto, foi constituído o Termo de Consentimento Livre e Esclarecido (TCLE) pelos pesquisadores. O estudo possui aprovação do Comitê de Ética e Pesquisa sob o parecer 3.908.788 e seguiu as diretrizes da resolução no 466/12 do Conselho Nacional de Saúde.

\section{Resultados}

Os dados descritos na Tabela 1 demonstram que foram avaliados, no total, 126 estudantes, sendo a maioria do sexo feminino $(60,3 \%)$, residentes com familiares $(79,4 \%)$ e que nunca se casaram $(95,2 \%)$. Além disso, $62,1 \%$ da amostra era da faixa etária de 20-35 anos, com uma média de 21 anos e 50\% se autodeclaram brancos. Ademais, grande parte dos alunos não possui profissão (96,8\%). Em relação aos aspectos da universidade, verificou-se que a maioria dos estudantes estava cursando o primeiro período $(57,1 \%)$ e não possui nenhuma forma de financiamento universitário, realizando o pagamento próprio das mensalidades $(60,3 \%)$. Outrossim, é que metade dos alunos possui renda maior que R\$10.000 (Tabela 1).

Tabela 1 - Caracterização sociodemográfica dos estudantes de medicina de uma universidade particular.

\begin{tabular}{|c|c|c|c|c|c|}
\hline Variáveis & \multirow{2}{*}{$\mathbf{n}^{\mathbf{o}}$} & \multirow{2}{*}{$\%$} & Variáveis & \multirow{2}{*}{$\mathbf{n}^{\mathbf{o}}$} & \multirow{2}{*}{$\%$} \\
\hline Sexo & & & Moradia & & \\
\hline Feminino & 76 & 60,3 & Sozinho & 22 & 17,5 \\
\hline Masculino & 50 & 39,7 & Com familiares & 100 & 79,4 \\
\hline Faixa etária & $\mathbf{n}^{\mathbf{o}}$ & $\%$ & República privada & 1 & 0,8 \\
\hline$<20$ & 44 & 35,5 & Outros & 3 & 2,4 \\
\hline Entre 20 e 35 & 77 & 62,1 & Profissão & $\mathbf{n}^{\mathbf{o}}$ & $\%$ \\
\hline$>35$ & 3 & 2,4 & Sim & 4 & 3,2 \\
\hline Estado civil & $\mathbf{n}^{\mathbf{o}}$ & $\%$ & Não & 122 & 96,8 \\
\hline Nunca se casou & 120 & 95,2 & Forma de custear o curso & $\mathbf{n}^{\mathbf{o}}$ & $\%$ \\
\hline Casado & 3 & 2,4 & FIES & 43 & 34,1 \\
\hline Divorciado & 2 & 1,6 & PROUNI & 4 & 3,2 \\
\hline Mora junto & 1 & 0,8 & Financiamento em banco privado & 1 & 0,8 \\
\hline Cor & $\mathbf{n}^{\mathbf{o}}$ & $\%$ & Pagamento próprio das mensalidades & 76 & 60,3 \\
\hline Branca & 63 & 50 & Outros & 2 & 1,6 \\
\hline Preta & 6 & 4,8 & Período & $\mathbf{n}^{\mathbf{o}}$ & $\%$ \\
\hline Parda & 56 & 44,4 & Primeiro & 72 & 57,1 \\
\hline \multirow{2}{*}{ Amarela } & \multirow{2}{*}{1} & \multirow{2}{*}{0,8} & Oitavo & 18 & 14,3 \\
\hline & & & Décimo Segundo & 36 & 28,6 \\
\hline
\end{tabular}

Fonte: Autores (2021).

No que se refere à qualidade de vida total e a análise dos domínios do Whoqol-bref - físico, psicológico, relações sociais e ambiente. A amostra populacional possui uma média de 58,9 na qualidade de vida total. Esse valor pode-se apresentar de 0 a 100, quanto maior o valor, melhor a qualidade de vida. Quando analisados os domínios, foi visto que o domínio de menor percentual foi o psicológico, com média de 64,2 e o maior, meio ambiente (70) (Tabela 2). 
Tabela 2 - Escore de qualidade de vida total e domínios.

\begin{tabular}{c|c|c}
\hline & Média & $\begin{array}{c}\text { Desvio } \\
\text { Padrão }\end{array}$ \\
\hline Físico & 65,3 & 15,4 \\
\hline Psicológico & 64,2 & 15,3 \\
\hline Relações Sociais & 69,1 & 20,1 \\
\hline Meio Ambiente & 70 & 14,6 \\
\hline Total & 58,9 & 8,4 \\
\hline
\end{tabular}

Fonte: Autores (2021).

Foi analisada a qualidade de vida dos alunos de acordo com o sexo. Em relação ao total, o sexo feminino apresentou uma média mais baixa $(58,6)$ do que o sexo masculino $(59,4)$. Além disso, as mulheres apresentam qualidade de vida abaixo dos homens em todos os domínios, exceto nas relações sociais. O pior domínio analisado em ambos os sexos foi o psicológico, com média 63,1 no sexo feminino e 65,8, no masculino. Entretanto, os valores dos domínios e o total deles não mostraram significância em relação ao sexo, pois todos os valores de p foram maiores que 0,05 (Tabela 3).

Quanto à QV, no primeiro período, a média total dos domínios foi de 59,8, com a maior qualidade de vida no domínio do meio ambiente $(72,5)$ e a menor média no domínio psicológico $(65,6)$. No oitavo período, a média total dos domínios foi de 54, com a maior qualidade de vida no domínio do meio ambiente (62) e a média mais baixa no domínio psicológico (56,3). Já no décimo segundo período, a média total dos domínios foi de 59,8, com a maior qualidade de vida no domínio das relações sociais (72) e a pior média no domínio psicológico $(65,3)$. No aspecto geral, o período em que os alunos apresentam a pior qualidade de vida em todos os domínios, é o oitavo. Já o primeiro e o décimo, apresentam números equivalentes, exceto no domínio do meio ambiente e relações sociais com maior diferença entre os valores. Os dados relevantes com valor de $\mathrm{p}>0,05$ foram encontrados no domínio psicológico, meio ambiente e média total da QV (Tabela 3).

Em relação à moradia, os estudantes que moram sozinhos apresentam como o pior domínio, o psicológico $(65,1)$ e o melhor, relações sociais (68), com um média total de 58,7, ficando abaixo somente dos que moram com os familiares $(59,4)$. Nesse último caso, os estudantes apresentam melhores números em todos os aspectos. Já os que moram em república privada apresentam o pior valor total, como pior domínio, o psicológico $(33,3)$ e o melhor, o físico. Além disso, somente os valores encontrados para o domínio do meio ambiente apresentam relevância no quesito moradia (Tabela 3).

$\mathrm{Na}$ questão da profissão, a média total foi maior naqueles que não possuem $(59,1)$. Além disso, esses alunos apresentam maiores valores que todos os domínios, sendo o meio ambiente, o melhor e o pior naqueles que possuem uma profissão. Os dados desse domínio são os únicos que apresentam relevância no quesito profissão (Tabela 3).

No que se refere a faixa etária, a qualidade de vida dos menores de 20 anos é superior às demais, no total e em todos os domínios, sendo o meio ambiente, o melhor (75,3) e o psicológico, o pior $(66,5)$. Já os alunos entre 20 e 35 anos, apresentam o total de 58, com o domínio de relações sociais com o maior valor $(68,2)$ e o menor, o psicológico $(63,3)$. Os maiores de 35 anos, apresentaram a pior qualidade de vida com o total de 48, e o domínio físico com o valor mais baixo (48,8). O domínio que apresentou valores relevantes foi o ambiental e o valor total da qualidade de vida (Tabela 3). 
Tabela 3 - Qualidade de vida e perfil sociodemográfico.

\begin{tabular}{|c|c|c|c|c|c|}
\hline Variáveis & \multirow{2}{*}{ Físico } & \multirow{2}{*}{ Psicológico } & \multirow{2}{*}{$\begin{array}{l}\text { Relações } \\
\text { Sociais }\end{array}$} & \multirow{2}{*}{$\begin{array}{c}\text { Meio } \\
\text { Ambiente }\end{array}$} & \multirow{2}{*}{ Total } \\
\hline Sexo, Média $(D P)$ & & & & & \\
\hline Feminino & $\begin{array}{c}64,2 \\
(15,7) \\
\end{array}$ & $63,1(16,6)$ & $69,4(19,8)$ & $69,8(15,9)$ & $58,6(8,6)$ \\
\hline Masculino & $66,9(15)$ & $65,8(13,4)$ & $68,5(20,8)$ & $70,3(12,8)$ & $59,4(8,2)$ \\
\hline p-valor ${ }^{\mathrm{M}}$ & 0,363 & 0,492 & 0,793 & 0,91 & 0,522 \\
\hline Estado civil, Média $(D P)$ & Físico & Psicológico & $\begin{array}{l}\text { Relações } \\
\text { Sociais }\end{array}$ & $\begin{array}{c}\text { Meio } \\
\text { Ambiente }\end{array}$ & Total \\
\hline Nunca se casou & $\begin{array}{c}65,9 \\
(15,1) \\
\end{array}$ & $64,3(15,3)$ & $69,3(20,2)$ & $70,7(14,2)$ & $59,2(8,2)$ \\
\hline Casado & $61,9(7,4)$ & $68,1(13,4)$ & $61,1(12,7)$ & $61,5(12,6)$ & $56,4(6,4)$ \\
\hline Separado/Divorciado & $50(35,4)$ & $58,3(29,5)$ & $75(35,4)$ & $57,8(28,7)$ & $\begin{array}{c}54,6 \\
(20,6) \\
\end{array}$ \\
\hline Mora junto & $42,9(0)$ & $45,8(0)$ & $58,3(0)$ & $37,5(0)$ & $45,5(0)$ \\
\hline p-valor ${ }^{K}$ & 0,425 & 0,659 & 0,631 & 0,193 & 0,443 \\
\hline Raça/cor, Média (DP) & Físico & Psicológico & $\begin{array}{l}\text { Relações } \\
\text { Sociais }\end{array}$ & $\begin{array}{c}\text { Meio } \\
\text { Ambiente } \\
\end{array}$ & Total \\
\hline Branca & $68,1(15)$ & $66,4(16,2)$ & $70,8(20)$ & $72,9(13,3)$ & $60,5(8,5)$ \\
\hline Preta & $67,4(6,9)$ & $56,3(16,8)$ & $66,7(21,1)$ & $60,2(20,5)$ & $55,7(6,4)$ \\
\hline Parda & $62,1(16)$ & $62,4(14,2)$ & $68(20)$ & $67,9(15,2)$ & $57,7(8,3)$ \\
\hline Amarela & $64,3(0)$ & $58,3(0)$ & $33,3(0)$ & $56,3(0)$ & $50(0)$ \\
\hline p-valor ${ }^{\mathrm{K}}$ & 0,27 & 0,244 & 0,393 & 0,101 & 0,125 \\
\hline Moradia, Média (DP) & Físico & Psicológico & $\begin{array}{l}\text { Relações } \\
\text { Sociais }\end{array}$ & $\begin{array}{c}\text { Meio } \\
\text { Ambiente }\end{array}$ & Total \\
\hline Sozinho & $\begin{array}{c}65,7 \\
(11,5) \\
\end{array}$ & $65,1(15,6)$ & $68(19,6)$ & $66,8(14,1)^{a, b}$ & $58,7(7,4)$ \\
\hline Com familiares & $\begin{array}{c}65,9 \\
(15,8) \\
\end{array}$ & $64,8(14,9)$ & $69,9(20,3)$ & $71,6(14,1)^{\mathrm{a}}$ & $59,4(8,4)$ \\
\hline República privada & $64,3(0)$ & $29,2(0)$ & $33,3(0)$ & $53,1(0)^{a, b}$ & $44,8(0)$ \\
\hline Outros & $44(19,7)$ & $50(15)$ & $61,1(12,7)$ & $46,9(16,2)^{b}$ & $48,3(10)$ \\
\hline p-valor ${ }^{\mathrm{K}}$ & 0,289 & 0,137 & 0,317 & 0,026 & 0,126 \\
\hline Profissão, Média (DP) & Físico & Psicológico & $\begin{array}{l}\text { Relações } \\
\text { Sociais } \\
\end{array}$ & $\begin{array}{c}\text { Meio } \\
\text { Ambiente } \\
\end{array}$ & Total \\
\hline Sim & $\begin{array}{c}57,1 \\
(24,4) \\
\end{array}$ & $59,4(17,1)$ & $60,4(15,8)$ & $53,9(13,4)$ & $\begin{array}{c}52,9 \\
(10,7) \\
\end{array}$ \\
\hline Não & $\begin{array}{c}65,6 \\
(15,1) \\
\end{array}$ & $64,3(15,3)$ & $69,4(20,3)$ & $70,5(14,4)$ & $59,1(8,3)$ \\
\hline p-valor ${ }^{\mathrm{M}}$ & 0,534 & 0,515 & 0,287 & 0,032 & 0,178 \\
\hline $\begin{array}{c}\text { Faixa Etária, Média } \\
(D P)\end{array}$ & Físico & Psicológico & $\begin{array}{l}\text { Relações } \\
\text { Sociais }\end{array}$ & $\begin{array}{c}\text { Meio } \\
\text { Ambiente } \\
\end{array}$ & Total \\
\hline$<20$ & $\begin{array}{c}68,1 \\
(12,5) \\
\end{array}$ & $66,5(12,2)$ & $72,3(19,2)$ & $75,3(12,3)^{\mathrm{a}}$ & $\begin{array}{c}61,2(6,6) \\
a\end{array}$ \\
\hline Entre 20 e 35 & $64(16,1)$ & $63,3(17)$ & $68,2(20,5)$ & $67,8(14,7)^{b}$ & $58(8,9)^{a, b}$ \\
\hline$>35$ & $\begin{array}{c}48,8 \\
(21,8) \\
\end{array}$ & $52,8(13,4)$ & $52,8(4,8)$ & $49(11)^{b}$ & $\begin{array}{c}48,5(7,5) \\
b\end{array}$ \\
\hline $\mathrm{p}$-valor ${ }^{\mathrm{K}}$ & 0,145 & 0,231 & 0,143 & 0,003 & 0,033 \\
\hline Período, Média (DP) & Físico & Psicológico & $\begin{array}{l}\text { Relações } \\
\text { Sociais } \\
\end{array}$ & $\begin{array}{c}\text { Meio } \\
\text { Ambiente } \\
\end{array}$ & Total \\
\hline Primeiro & $\begin{array}{c}66,2 \\
(14,1) \\
\end{array}$ & $65,6(14,1)^{\mathrm{a}}$ & $69,8(21,1)$ & $72,5(13,4)^{\mathrm{a}}$ & $59,8(7,8)^{\mathrm{a}}$ \\
\hline
\end{tabular}




\begin{tabular}{c|c|c|c|c|c}
\hline Oitavo & $\begin{array}{c}59,1 \\
(14,7)\end{array}$ & $\begin{array}{c}56,3(13,6) \\
\mathrm{b}\end{array}$ & $60,2(20,5)$ & $62(16,3)^{\mathrm{b}}$ & $54(8)^{\mathrm{b}}$ \\
\hline Décimo Segundo & $\begin{array}{c}66,8 \\
(17,8)\end{array}$ & $\begin{array}{c}65,3(17,5) \\
\mathrm{a}, \mathrm{b}\end{array}$ & $72(17,2)$ & $69,4(14,8)^{\mathrm{a}, \mathrm{b}}$ & $\begin{array}{c}59,8(9,1) \\
\mathrm{a}, \mathrm{b}\end{array}$ \\
\hline p-valor $^{\mathrm{K}}$ & 0,166 & 0,042 & 0,112 & 0,04 & 0,032 \\
\hline
\end{tabular}

Legenda: DP - Desvio Padrão. R - Correlação de Spearman. M - Teste de Mann-Whitney. K - Teste de Kruskal-Wallis. a,b Subgrupos distintos ao nível de 5\% para o teste de Mann-Whitney- Bonferroni.

Fonte: Autores (2021).

Sob o parâmetro da forma de custeio do curso, os alunos que possuem PROUNI apresentam os piores parâmetros em todos os domínios, exceto em relações sociais, na qual a categoria "outros" apresenta o pior valor $(41,7)$. Estudantes que possuem FIES apresentam o valor mais alto em relações sociais $(69,4)$ e o mais baixo no aspecto psicológico. Ademais, a média total mais alta $(66,6)$ está com os alunos que fazem financiamento em banco privado, os quais apresentem o meio ambiente como melhor domínio $(84,4)$. Em relação aos alunos que fazem o pagamento próprio das mensalidades, apresentam a melhor média em meio ambiente $(71,8)$ e a pior no aspecto psicológico $(64,4)$. Nenhum valor no aspecto de forma de custeio valor foi relevante, pois todos apresentaram valor de $\mathrm{p}<0,05$ (Tabela 4).

Além disso, foram observados valor relevantes em alguns questionamentos quanto aos alunos que realizam financiamento, como: prejuízo em seu desempenho acadêmico por preocupar-se com o pagamento futuro do financiamento, se sente prejuízo em sua qualidade de vida por preocupar-se com o pagamento futuro do financiamento, e se o aluno, com que frequência, sente-se preocupado/ansioso por não saber se conseguirá concluir seu curso por motivos financeiros. Em relação ao desempenho acadêmico no domínio social mostrou uma maior diferença entre aqueles que responderam sim e não. No quesito prejuízo da qualidade de vida, o domínio psicológico e meio ambiente foram os mais discrepantes. No questionamento sobre preocupação para concluir o curso, a diferença entre os alunos que responderam nunca e sempre ficou mais evidente no domínio de relações sociais (Tabela 4).

Em relação aos alunos que realizaram o pagamento próprio das mensalidades foram observados valor significantes em alguns questionamentos quanto aos alunos que realizam financiamento, como no prejuízo de desempenho acadêmico e na qualidade de vida. Em relação ao desempenho acadêmico e prejuízo na qualidade de vida, o domínio do meio ambiente mostrou uma maior diferença entre aqueles que responderam sim e não (Tabela 4).

Tabela 4 - Qualidade de vida e questionário sobre forma de custeio.

\begin{tabular}{|c|c|c|c|c|c|}
\hline Forma de custear o curso, Média (DP) & Físico & Psicológico & $\begin{array}{c}\text { Relações } \\
\text { Sociais }\end{array}$ & $\begin{array}{c}\text { Meio } \\
\text { Ambiente }\end{array}$ & Total \\
\hline FIES & $65,5(16,9)$ & $63,4(17,3)$ & $69,4(19,6)$ & $68,8(13,8)$ & $58,7(8,7)$ \\
\hline PROUNI & $50,9(21,1)$ & $61,5(18,8)$ & $62,5(10,8)$ & $46,9(22,7)$ & $51,5(9,9)$ \\
\hline Financiamento em Banco Privado & $82,1(0)$ & $75(0)$ & $75(0)$ & $84,4(0)$ & $66,6(0)$ \\
\hline $\begin{array}{l}\text { Sem financiamento, pagamento próprio das } \\
\text { mensalidades }\end{array}$ & $65,9(14)$ & $64,4(14,4)$ & $69,9(20,8)$ & $71,8(13,9)$ & $59,4(8,2)$ \\
\hline Outros & $62,5(22,7)$ & $70,8(0)$ & $41,7(0)$ & $70,3(6,6)$ & $55,3(2,6)$ \\
\hline p-valor ${ }^{\mathrm{K}}$ & 0,446 & 0,877 & 0,303 & 0,154 & 0,367 \\
\hline $\begin{array}{l}\text { Com que frequência se sente preocupado/ansioso } \\
\text { quanto ao pagamento futuro do financiamento, } \\
\text { Média }(D P)\end{array}$ & Físico & Psicológico & $\begin{array}{c}\text { Relações } \\
\text { Sociais }\end{array}$ & $\begin{array}{c}\text { Meio } \\
\text { Ambiente }\end{array}$ & Total \\
\hline Nunca & $70,2(12,6)$ & $69,9(12,8)$ & $81,5(8,1)$ & $71,5(7,6)$ & $62,9(4,9)$ \\
\hline Algumas vezes & $69,7(16,4)$ & $66,3(18,7)$ & $71,1(17,7)$ & $74,3(13,2)$ & $61(7,9)$ \\
\hline Frequentemente & $66,5(16,5)$ & $67,4(12,3)$ & $68,8(15,9)$ & $67,6(10)$ & $59,2(7,1)$ \\
\hline
\end{tabular}




\begin{tabular}{|c|c|c|c|c|c|}
\hline Muito frequente & $67,9(18,9)$ & $68,1(12,7)$ & $61,1(21)$ & $67,7(18,3)$ & $58,4(8,5)$ \\
\hline Sempre & $50,7(15,3)$ & $50,9(18,5)$ & $55,6(26)$ & $60,4(17,7)$ & $50,8(9,9)$ \\
\hline $\mathrm{p}$-valor ${ }^{\mathrm{K}}$ & 0,09 & 0,207 & 0,092 & 0,238 & 0,064 \\
\hline $\begin{array}{l}\text { Sente prejuízo em seu desempenho acadêmico por } \\
\text { preocupar-se com o pagamento futuro do } \\
\text { financiamento, Média }(D P)\end{array}$ & Físico & Psicológico & $\begin{array}{l}\text { Relações } \\
\text { Sociais }\end{array}$ & $\begin{array}{c}\text { Meio } \\
\text { Ambiente }\end{array}$ & Total \\
\hline Sim & $49,3(15,9)$ & $48,8(18,9)$ & $51,2(27,8)$ & $56,7(15,5)$ & $49(9,5)$ \\
\hline Não & $68,3(15,3)$ & $67,1(15,4)$ & $72,2(15,9)$ & $71,7(12,1)$ & $60,7(7,1)$ \\
\hline p-valor ${ }^{\mathrm{M}}$ & 0,008 & 0,013 & 0,038 & 0,02 & 0,005 \\
\hline $\begin{array}{l}\text { Sente prejuízo em sua qualidade de vida por } \\
\text { preocupar-se com o pagamento futuro do } \\
\text { financiamento, Média }(D P)\end{array}$ & Físico & Psicológico & $\begin{array}{l}\text { Relações } \\
\text { Sociais }\end{array}$ & $\begin{array}{c}\text { Meio } \\
\text { Ambiente }\end{array}$ & Total \\
\hline Sim & $59,8(17,3)$ & $56,2(16,8)$ & $62,2(21,4)$ & $61,3(12,5)$ & $54,3(8,7)$ \\
\hline Não & $67,6(16,2)$ & $67,5(16,3)$ & $71,7(18,2)$ & $72,6(12,8)$ & $60,7(7,9)$ \\
\hline p-valor ${ }^{M}$ & 0,221 & 0,038 & 0,145 & 0,009 & 0,013 \\
\hline $\begin{array}{l}\text { Com que frequência se sente preocupado/ansioso } \\
\text { por não saber se conseguirá concluir seu curso por } \\
\text { motivos financeiros, Média }(D P)\end{array}$ & Físico & Psicológico & $\begin{array}{l}\text { Relações } \\
\text { Sociais }\end{array}$ & $\begin{array}{c}\text { Meio } \\
\text { Ambiente }\end{array}$ & Total \\
\hline Nunca & $72,1(13,8)^{\mathrm{a}}$ & $69,8(11,5)$ & $76(19,1)$ & $77,9(12,6)^{\mathrm{a}}$ & $\begin{array}{c}63,3(6,9) \\
\mathrm{a}\end{array}$ \\
\hline Algumas vezes & $\begin{array}{c}63,9(12,3) \\
\mathrm{a}, \mathrm{b}\end{array}$ & $66,3(12,3)$ & $70,2(19,4)$ & $73(12,4)^{a, b}$ & $\begin{array}{c}59,7(7,2) \\
a, b\end{array}$ \\
\hline Frequentemente & $68,1(8,3)^{a, b}$ & $60,1(15,3)$ & $62,5(22,7)$ & $66,7(13,2)^{a, b, c}$ & $\begin{array}{c}56,5(6,6) \\
\mathrm{b}\end{array}$ \\
\hline Muito frequente & $62(6,7)^{a, b}$ & $57(16)$ & $67,4(14,5)$ & $64,1(7,1)^{b, c}$ & $\begin{array}{c}55,8(3,8) \\
\mathrm{b}\end{array}$ \\
\hline Sempre & $\begin{array}{c}55,4(20,3) \\
\mathrm{b}\end{array}$ & $54,2(18,4)$ & $54,7(27,4)$ & $59(16,3)^{\mathrm{c}}$ & $\begin{array}{c}51,7 \\
(11,5)^{\mathrm{b}}\end{array}$ \\
\hline $\mathrm{p}$-valor ${ }^{\mathrm{K}}$ & 0,035 & 0,094 & 0,203 & 0,005 & 0,01 \\
\hline $\begin{array}{l}\text { Com que frequência se sente preocupado/ansioso } \\
\text { quanto ao pagamento próprio, Média }(D P)\end{array}$ & Físico & Psicológico & $\begin{array}{l}\text { Relações } \\
\text { Sociais }\end{array}$ & $\begin{array}{c}\text { Meio } \\
\text { Ambiente }\end{array}$ & Total \\
\hline Nunca & $73,7(13,6)^{\mathrm{a}}$ & $73,5(8,4)$ & $77,3(19,4)$ & $73,3(16,3)$ & $63,6(7,9)$ \\
\hline Algumas vezes & $\underset{\mathrm{a}, \mathrm{b}}{69,3(12,8)}$ & $65,6(14,7)$ & $73,6(18,5)$ & $74,6(11,1)$ & $61,2(7,3)$ \\
\hline Frequentemente & $\underset{\mathrm{a}, \mathrm{b}}{63,7(10,4)}$ & $65,1(12,1)$ & $64,6(23,1)$ & $74(13,1)$ & $58,8(7)$ \\
\hline Muito frequente & $\underset{\mathrm{a}, \mathrm{b}}{67,4(11,8)}$ & $66,7(13)$ & $70,4(20,3)$ & $73,4(12,6)$ & $60,5(6,8)$ \\
\hline Sempre & $\begin{array}{c}58,6(15,4) \\
\mathrm{b}\end{array}$ & $56,1(16,5)$ & $61,9(23,2)$ & $62,5(14,3)$ & $54,3(8,8)$ \\
\hline $\mathrm{p}$-valor ${ }^{\mathrm{K}}$ & 0,048 & 0,067 & 0,391 & 0,14 & 0,078 \\
\hline $\begin{array}{l}\text { Sente prejuízo em seu desempenho acadêmico por } \\
\text { preocupar-se com o custeio mensal do seu curso, } \\
\text { Média }(D P)\end{array}$ & Físico & Psicológico & $\begin{array}{l}\text { Relações } \\
\text { Sociais }\end{array}$ & $\begin{array}{c}\text { Meio } \\
\text { Ambiente }\end{array}$ & Total \\
\hline Sim & $59,3(14,1)$ & $55,1(17,5)$ & $59,2(18,9)$ & $59,6(10,5)$ & $53,3(7,2)$ \\
\hline Não & $68,4(13,2)$ & $67,3(12,7)$ & $72,6(21,2)$ & $75,4(13,1)$ & $61,4(7,7)$ \\
\hline p-valor ${ }^{\mathrm{M}}$ & 0,026 & 0,015 & 0,022 & $<0,001$ & 0,001 \\
\hline
\end{tabular}




\begin{tabular}{l|c|c|c|c|c}
\hline $\begin{array}{l}\text { Sente prejuízo em sua qualidade de vida por } \\
\text { preocupar-se com o custeio mensal do seu curso, } \\
\text { Média }(D P)\end{array}$ & Físico & Psicológico & $\begin{array}{c}\text { Relaç̃̃es } \\
\text { Sociais }\end{array}$ & $\begin{array}{c}\text { Meio } \\
\text { Ambiente }\end{array}$ & Total \\
\hline Sim & $60,5(13,9)$ & $58,9(16)$ & $62,2(21,1)$ & $64,6(11,6)$ & $55,4(7,9)$ \\
\hline Não & $70,7(12,4)$ & $68,9(12,3)$ & $73,6(19,5)$ & $76,8(13,4)$ & $62,6(7,1)$ \\
\hline p-valor ${ }^{\mathrm{M}}$ & 0,003 & 0,016 & 0,02 & $<0,001$ & 0,001 \\
\hline
\end{tabular}

Legenda: DP - Desvio Padrão. R - Correlação de Spearman. M - Teste de Mann-Whitney. K - Teste de Kruskal-Wallis. a,b Subgrupos distintos ao nível de 5\% para o teste de Mann-Whitney- Bonferroni.

Fonte: Autores (2021).

\section{Discussão}

Um trabalho realizado em uma universidade na África do Sul mostrou valores mais baixos nos domínios físico, psicológico e meio ambiente em estudantes do quarto ano da graduação (Colby et al., 2018). Em um estudo sistemático que observou fatores relacionados à qualidade de vida em estudantes de medicina brasileiros mostrou que estar no terceiro e sexto ano da graduação são fatores relacionados à pior qualidade de vida (Solis \& Lotufo-Neto, 2019).

No presente trabalho foi observado que os alunos do oitavo período, ou seja, que estão no quarto ano da graduação possuem médias em todos os domínios mais baixas, principalmente, no psicológico, mas sem valores significativos. Um estudo realizado na faculdade de São Paulo mostrou que o primeiro e segundo ano de faculdade possuem uma percepção mais positiva do aluno do que os quatro últimos anos. Esses dados foram associados a alta carga horária e a maior responsabilidade do aluno nesses últimos anos de graduação (Enns et al., 2016).

Já em um estudo realizado na universidade de São Paulo não encontrou diferença na qualidade de vida em relação ao período ou ano de graduação (Serinolli \& Novaretti, 2017). Outro estudo realizado na Arabia Saudita (Malibary et al., 2019) e em uma universidade particular do Paquistão (Aziz et al., 2020) também corroboraram com a indiferença da QV com o período do curso.

Em um estudo sistemático foi observado que estudantes com financiamento, especificamente o Fundo de Financiamento Estudantil (FIES), possuem pior qualidade de vida (Solis \& Lotufo-Neto, 2019). Nessa pesquisa foi mostrado também que os estudantes com financiamento FIES e Programa Universidade para Todos (PROUNI) possuem piores valores em todos os domínios do questionário. Dados que coincidem com o presente estudo, em que os alunos que possuem financiamento têm menor média em todos os domínios, exceto relações sociais. Além disso, aqueles que possuem PROUNI apresentam a pior média. Outrossim, é que aqueles com financiamento afirmam ter pior desempenho e qualidade de vida comparado com os que fazem pagamento próprio das mensalidades.

Segundo Lins et al. (2015), os alunos com FIES possuíam menor qualidade de vida relacionada à saúde.

O financiamento, FIES, varia de $50 \%$ a $100 \%$ das mensalidades dependendo da renda familiar per capita. A taxa de juros é de 3,4\% por ano e possui 18 meses de carência após o prazo do financiamento (Fundo de Financiamento ao Estudante do Ensino Superior, n.d.). Dessa forma, os alunos acumulam dívidas durante o curso, o que leva a um estresse adicional que pode afetar a qualidade de vida.

De acordo com um estudo feito na faculdade de medicina 9 de julho, os estudantes que já possuíam uma graduação não tinham qualidade de vida diferente daqueles sem graduação (Serinolli \& Novaretti, 2017).

Um estudo realizado na Alemanha, indicou que aqueles que dividem seu tempo entre trabalho e estudo, possuem mais estresse e pior desempenho acadêmico, já que possuem menos tempo para estudar. Além disso, é desafiador para os alunos encontrar um trabalho que encaixe no currículo acadêmico que muitas vezes é inflexível (Bergmann et al., 2019). Em outro trabalho alemão com alunos no primeiro ano da graduação, foi observado que aqueles que trabalhavam meio turno, ou turno 
completo em comparação aqueles que não têm emprego, não foi observado diferença significativa na qualidade de vida (Heinen et al., 2017).

Já em uma pesquisa realizada na universidade de medicina de Tabriz, Irã, observou que os alunos que possuíam emprego tiveram pior qualidade de vida do que aqueles que não, com valores significantes. As pessoas empregadas tinham média de qualidade de vida 50,2 e as que não possuíam emprego tinham média de 65.4 (Ghassab-Abdollahi et al., 2020). No presente estudo foi observado que pessoas que possuem uma profissão têm pior qualidade de vida, comparada com aquelas que não. Isso pode ser justificado pela carga horária extensa entre trabalho e disciplinas da faculdade.

Em outra pesquisa realizada nos Estados Unidos que analisou estudantes de medicina através de questionário eletrônico, o Medical Student Wellbeing Index. Observou-se que os estudantes com 28 anos ou mais possuíam maior probabilidade de ter sofrimento psíquico severo (Rajapuram et al., 2020). Corroborando com esta pesquisa, no presente estudo foi demonstrado dados parecidos com aqueles que tinham idade maior que 35 anos, os quais possuem piores médias no domínio do meio ambiente do que aqueles com menor idade.

Foi observado em um estudo no Irã, na universidade de Tabriz (Ghassab-Abdollahi et al., 2020) que os alunos em moradia alugada apresentaram pior qualidade de vida, já os que tiveram maior QV foram os com casa própria (média de 70.9). Outro trabalho que corrobora com esses resultados foi realizado na Universidade Federal de São Paulo, mostrando que os alunos que moravam sozinho, comparados àqueles que moravam com o cônjuge, pais, irmãos, parentes ou amigos, apresentaram menores escores no domínio físico (Cunha et al., 2017).

Outro estudo realizado em Portugal mostrou que os alunos que saíram da casa da família apresentavam níveis baixos de qualidade de vida em todos os domínios do que aqueles que moravam com a família (Vizzotto et al., 2017). Entretanto, em uma pesquisa realizada no Iêmen, mostrou que os alunos moram em casa de família tem menor QV no domínio físico e em relações sociais quando comparados aos que moram em dormitórios, com valores significantes (Obad et al., 2021).

Já no presente estudo, os alunos com melhor qualidade de vida eram aqueles que moravam com os pais $(71,6)$ e em segundo lugar aqueles que moravam sozinhos (66,8), no domínio meio ambiente. Segundo Tassini et al. (2017), as mudanças na vida psicossocial, como o afastamento do seio familiar contribui para uma piora no estilo de vida.

Em um estudo no estado de São Paulo, Brasil, mostrou que o sexo feminino possui pior qualidade de vida quando comparado ao masculino, principalmente nos domínios físico, psicológico e social, porém sem valores significativos estatisticamente (Serinolli \& Novaretti, 2017). No presente estudo foram observados dados parecidos, apesar da ausência de significância.

De acordo com um estudo na Arabia Saudita (Malibary et al., 2019) e no Paquistão (Aziz et al., 2020) não houve diferença significante de qualidade de vida entre homens e mulheres. Já em um trabalho realizado na Malásia observou que as estudantes (66.32) possuem menor QV no domínio psicológico comparado aos estudantes (70,73) com valores significantes (Gan \& Yuen Ling, 2019). Outro estudo realizado no país de Trindade e Tobago mostrou que no domínio social e no meio ambiente as mulheres possuem menor qualidade de vida (Chattu et al., 2020).

Além disso, um estudo de revisão e meta-analise observou que o sexo feminino está associado a depressão, ansiedade e estresse (Pacheco et al., 2017).

\section{Conclusão}

De acordo com o estudo fica notório que há prejuízo na qualidade de vida dos estudantes de medicina relacionado a fatores sociodemográficos. Entretanto, na literatura há divergência entre os principais aspectos influenciadores, por conta das características populacionais de cada estudo. Dessa forma, são necessários maiores estudos a fim de estabelecer melhores 
indicativos das variáveis sociodemográficas, e a partir disso definir estratégias que possam minimizar o impacto na qualidade de vida.

O curso de medicina pode interferir negativamente na qualidade de vida dos estudantes de medicina, principalmente nos que moram em república privada, maiores de 35 anos, naqueles que têm financiamento do curso e nos que possuem profissão. De uma maneira geral, os domínios mais prejudicados são o físico e o psicológico. Além disso, a literatura mostra que outros fatores podem ser indicativos relevantes no prejuízo na qualidade de vida, como: estar nos últimos anos da faculdade e ser do sexo feminino.

Dessa forma, é de extrema importância que a universidade ofereça e incentive atividades de lazer a fim de contribuir com a qualidade de vida. Ademais, com a finalidade de melhorar o domínio psicológico dos estudantes, deve-se manter, divulgar e incentivar o Núcleo de Apoio Pedagógico e Psicossocial (NAPPS), com o objetivo de que mais estudantes possam buscar apoio para as questões pessoais enfrentadas durante a graduação.

\section{Referências}

Amorim, B. B., Moraes, L., Sá, I. C. G., Silva, B. B. G., \& Camara Filho, J. W. S. (2018). Saúde Mental do Estudante de Medicina: Psicopatologia, Estresse, Sono e Qualidade de Vida. Revista Psicologia, Diversidade e Saúde, 7(2), 245-254. 10.17267/2317-3394rpds.v7i2.1911.

Aziz, Y., Khan, A. Y., Shahid, I., Khan, M. A. M., \& Aisha, A. (2020). Quality of life of students of a private medical college. Pakistan Journal of Medical Sciences, 36(2), Article 2. https://doi.org/10.12669/pjms.36.2.668.

Bergmann, C., Muth, T., \& Loerbroks, A. (2019). Medical students' perceptions of stress due to academic studies and its interrelationships with other domains of life: A qualitative study. Medical Education Online, 24(1), 1603526. https://doi.org/10.1080/10872981.2019.1603526.

Chattu, V. K., Sahu, P. K., Seedial, N., Seecharan, G., Seepersad, A., Seunarine, M., Sieunarine, S., Seymour, K., Simboo, S., \& Singh, A. (2020). An Exploratory Study of Quality of Life and Its Relationship with Academic Performance among Students in Medical and other Health Professions. Medical Sciences, 8(2), 23. https://doi.org/10.3390/medsci8020023.

Chazan, A. C. S., Campos, M. R., \& Portugal, F. B. (2015). Qualidade de vida de estudantes de medicina da UERJ por meio do Whoqol-bref: Uma abordagem multivariada. Ciência \& Saúde Coletiva, 20, 547-556. https://doi.org/10.1590/1413-81232015202.05182014.

Colby, L., Mareka, M., Pillay, S., Sallie, F., van Staden, C., du Plessis, E. D., \& Joubert, G. (2018). The association between the levels of burnout and quality of life among fourth-year medical students at the University of the Free State. The South African Journal of Psychiatry: SAJP: The Journal of the Society of Psychiatrists of South Africa, 24, 1101. https://doi.org/10.4102/sajpsychiatry.v24i0.1101.

Cunha, D. H. F. da, Moraes, M. A. de, Benjamin, M. R., \& Santos, A. M. N. dos. (2017). Percepção da qualidade de vida e fatores associados aos escores de qualidade de vida de alunos de uma escola de medicina. Jornal Brasileiro de Psiquiatria, 66, 189-196. https://doi.org/10.1590/0047-2085000000170.

Enns, S. C., Perotta, B., Paro, H. B., Gannam, S., Peleias, M., Mayer, F. B., Santos, I. S., Menezes, M., Senger, M. H., Barelli, C., Silveira, P. S. P., Martins, M. A., \& Zen Tempski, P. (2016). Medical Students' Perception of Their Educational Environment and Quality of Life: Is There a Positive Association? Academic Medicine, 91(3), 409-417. https://doi.org/10.1097/ACM.0000000000000952.

Fundo De Financiamento Ao Estudante Do Ensino Superior. ([s.d.]). https://sisfiesportal.mec.gov.br/.

Gan, G. G., \& Yuen Ling, H. (2019). Anxiety, depression and quality of life of medical students in Malaysia. The Medical Journal of Malaysia, 74(1), 57-61.

Ghassab-Abdollahi, N., Shakouri, S. K., Aghdam, A. T., Farshbaf-Khalili, A., Abdolalipour, S., \& Farshbaf-Khalili, A. (2020). Association of quality of life with physical activity, depression, and demographic characteristics and its predictors among medical students. Journal of Education and Health Promotion, 9 , 147. https://doi.org/10.4103/jehp.jehp_91_20.

Heinen, I., Bullinger, M., \& Kocalevent, R.-D. (2017). Perceived stress in first year medical students-Associations with personal resources and emotional distress. BMC Medical Education, 17(1), 4. https://doi.org/10.1186/s12909-016-0841-8.

Hwang, I. C., Park, K. H., Kim, J. J., Yim, J., Ko, K. P., Bae, S. M., \& Kyung, S. Y. (2017). Perceived Social Support as a Determinant of Quality of Life Among Medical Students: 6-Month Follow-up Study. Academic Psychiatry, 41(2), 180-184. https://doi.org/10.1007/s40596-016-0503-5.

Irribarra T., L., Mery I., P., Lira S., M. J., Campos D., M., González L., F., \& Irarrázaval D., S. (2018). ¿Cómo es la calidad de vida reportada por los estudiantes de Medicina? Revista médica de Chile, 146(11), 1294-1303. https://doi.org/10.4067/S0034-98872018001101294.

Lins, L., Carvalho, F. M., Menezes, M. S., Porto-Silva, L., \& Damasceno, H. (2015). Health-related quality of life of students from a private medical school in Brazil. International Journal of Medical Education, 6, 149-154. https://doi.org/10.5116/ijme.563a.5dec.

Malibary, H., Zagzoog, M. M., Banjari, M. A., Bamashmous, R. O., \& Omer, A. R. (2019). Quality of Life (QoL) among medical students in Saudi Arabia: A study using the WHOQOL-BREF instrument. BMC Medical Education, 19(1), 344. https://doi.org/10.1186/s12909-019-1775-8.

Meira, F., Moreno, G., Arruda, R. B. P. de, \& Veras, A. B. (2017). Nuances do conceito de saúde mental e a qualidade de vida de estudantes de medicina. Revista Sul-Americana de Psicologia, 5(2), 263-280. 
Obad, A. S., Abdulwali, F. K., Alaidroos, H. A. H., BaAbbad, A. A., Al-Gunaid, M. A., Al Ghurabi, M. O. S., Bawazir, A. A., BaFadhl, Z. A. A., Baqashmer, Z. A. A., \& Shareef, M. A. (2021). Relationship between shortage of basic life needs and quality of life of medical students in Yemen: A study utilizing validity and reliability of WHOQOL-BREF questionnaire. Journal of Family Medicine and Primary Care, 10(3), 1466-1472. https://doi.org/10.4103/jfmpc.jfmpc_935_20.

Oliveira, A. B. D. de. (2018). Estresse, síndrome de burnout e qualidade de vida em estudantes de medicina que utilizam metodologia ativa de ensinoaprendizagem: Um estudo transversal. https://ri.ufs.br/jspui/handle/riufs/7563.

Pacheco, J. P., Giacomin, H. T., Tam, W. W., Ribeiro, T. B., Arab, C., Bezerra, I. M., \& Pinasco, G. C. (2017). Mental health problems among medical students in Brazil: A systematic review and meta-analysis. Brazilian Journal of Psychiatry, 39(4), 369-378. https://doi.org/10.1590/1516-4446-2017-2223.

Rajapuram, N., Langness, S., Marshall, M. R., \& Sammann, A. (2020). Medical students in distress: The impact of gender, race, debt, and disability. PLOS ONE, 15(12), e0243250. https://doi.org/10.1371/journal.pone.0243250.

Scholz, M., Neumann, C., Steinmann, C., Hammer, C. M., Schröder, A., Eßel, N., Paulsen, F., \& Burger, P. H. M. (2015). [Development and correlation of work-related behavior and experience patterns, burnout and quality of life in medical students from their freshmanship to the first state examination]. Psychotherapie, Psychosomatik, Medizinische Psychologie, 65(3-4), 93-98. https://doi.org/10.1055/s-0034-1375630.

Serinolli, M. I., \& Novaretti, M. C. Z. (2017). A cross-sectional study of sociodemographic factors and their influence on quality of life in medical students at Sao Paulo, Brazil. PLOS ONE, 12(7), e0180009. https://doi.org/10.1371/journal.pone.0180009.

Solis, A. C., \& Lotufo-Neto, F. (2019). Predictors of quality of life in Brazilian medical students: A systematic review and meta-analysis. Brazilian Journal of Psychiatry, 41(6), 556-567. https://doi.org/10.1590/1516-4446-2018-0116.

Tassini, C. C., Val, G. R. do, Candido, S. da S., \& Bachur, C. K. (2017). Avaliação do Estilo de Vida em Discentes Universitários da Área da Saúde através do Questionário Fantástico. International Journal of Cardiovascular Sciences, 30, 117-122. https://doi.org/10.5935/2359-4802.20170024.

The World Health Organization Quality of Life assessment (WHOQOL): Position paper from the World Health Organization. (1995). Social Science \& Medicine (1982), 41(10), 1403-1409. https://doi.org/10.1016/0277-9536(95)00112-k.

Vizzotto, M. M., Jesus, S. N. de, \& Martins, A. C. (2017). Saudades de Casa: Indicativos de depressão, ansiedade, qualidade de vida e adaptação de estudantes universitários. Revista Psicologia e Saúde. https://doi.org/10.20435/pssa.v9i1.469. 